\title{
The Countability of a Tiling Family and the Periodicity of a Tiling*
}

\author{
N. Dolbilin \\ Steklov Institute, 117333 Moscow, Russia
}

\begin{abstract}
If a given finite protoset, together with a given finite matching rule, gives rise to at most countably many different tilings of $d$-dimensional space, then at least one of them is periodic.
\end{abstract}

\section{Introduction}

Danzer proved the following theorem: Given a finite matching rule $\mathscr{R}$, if precisely one Delone graph (up to congruence) fulfilling $\mathscr{R}$ exists, then this graph is periodic. In March 1991 at the Bielefeld workshop on Quasicrystals Marjorie Senechal raised a question: what answer should be expected if there were finitely many different Delone graphs. This question stimulated my efforts in a direction which has led to the theorem that we prove here. Peter Schmitt gave me an example of a protoset and a matching rule that induced finitely many (in Schmitt's case there were three) noncongruent tilings, among which periodic as well as nonperiodic occur. Alternatively, more concretely, two of his two-dimensional tilings happened to have twodimensional translational symmetry and the third one had only a one-dimensional translation group. So, if noncongruent tilings are finitely or countably many, then such protosets and matching rules that give periodic tilings can be pointed out along with weakly periodic and purely nonperiodic tilings.

In this paper we investigate the situation where a local rule $\mathscr{R}$ gives rise to finite or countable sets of tilings. To be precise, we consider tilings of $d$-dimensional space by compact polytopes. The polyhedral tilings should be considered as a more special case of the rather general concept of the Delone graph introduced by Danzer.

* This research was supported in part by the SFB 343 "Diskrete Strukturen in der Mathematik" at Bielefeld University and the Russian Foundation for Fundamental Research. 
However, in fact, it should be easily possible to formulate and to prove Theorem 1.1 in terms of Delone graphs.

Let us recall some basic definitions. A protoset $\mathscr{P}=\left\{P_{1}, \ldots, P_{n}\right\}$ is a family of compact polytopes (called prototiles) in $d$-dimensional euclidean (or hyperbolic) space $\mathbf{R}^{d}$. The prototiles can be thought of as being topological balls.

A matching rule $\mathscr{R}$ for a protoset $\mathscr{P}$ is a set of "rules" that determine, for each fixed copy $P$ of some prototile in $\mathscr{P}$, how $P$ may be surrounded by other copies of prototiles. A matching rule $\mathscr{R}$ is called finite if it allows each such set $P$ to be surrounded in only a finite number of different ways (considering only those polytopes that share boundary points with $P$ ). In this paper we only consider finite matching rules.

Given a protoset $\mathscr{P}$ and a matching rule $\mathscr{R}$, let $T$ be a family of polytopes, each congruent to some prototile contained in $\mathscr{P}$, and assume that $T$ obeys the matching rule $\mathscr{R}$. If $T$ tiles $\mathbf{R}^{d}$, then $T$ is called a tiling with protoset $\mathscr{P}$ and matching rule $\mathscr{R}$. The elements of $T$ are called tiles.

A tiling $T$ with protoset $\mathscr{P}$ and matching rule $\mathscr{R}$ is called periodic if its symmetry group $\Gamma_{T}$ (i.e., the group of all isometries of $\mathbf{R}^{d}$ that map $T$ onto $T$ ) possesses a compact fundamental domain, or, equivalently, partitions $\mathscr{T}$ into only finitely many $\Gamma_{T}$-equivalence classes.

Here it should be mentioned that in the euclidean space case, as a consequence of the Bieberbach-Schoenflis theorem, a translational subgroup of a finite index exists in such a group. So, our definition of a periodic tiling in euclidean space means standard generally accepted translational full-dimensional periodicity.

Theorem 1.1. Given a protoset $\mathscr{P}$ and a matching rule $\mathscr{R}$. Let $\mathscr{T}(\mathscr{P}, \mathscr{R})$ denote the family of all (congnuence classes of) tilings with protoset $\mathscr{P}$ and matching rule $\mathscr{R}$. Suppose that family $\mathscr{T} \mathscr{P}, \mathscr{R})$ is not empty. If $\mathscr{T} \mathscr{P}, \mathscr{R})$ is either finite or countably infinite, then at least one tiling $T \in \mathscr{T}(\mathscr{P}, \mathscr{R})$ that is periodic exists.

\section{Proof in Seven Lemmas}

To prove Theorem 1.1 we need a series of definitions and lemmas.

Given a tiling $T$, any two polytopes $P, P^{\prime} \in T$ can be connected by a chain $\left[P, P^{\prime}\right]$, which is a sequence $\left(P_{i_{0}}(=P), P_{i_{1}}, \ldots, P_{i_{m}}\left(=P^{\prime}\right)\right), P_{i_{j}} \in T$, in which any two neighboring elements $P_{i_{j-1}}, P_{i}, j \in\{1, \ldots, m\}$, have some nonempty part of their boundaries in common. The number $m$ is called the length of the chain. The length of the shortest chain connecting two polytopes $P$ and $P^{\prime}$ is called the distance $\left\|P, P^{\prime}\right\|$ between $P$ and $P^{\prime}$.

Given $k \in \mathbf{N}$ and $P \in T$, the subcomplex $\mathscr{C}^{i}(P)$ of the tiling $T$ defined as

$$
\mathscr{C}^{i}(P):=\left\{P^{\prime} \mid\left\|P, P^{\prime}\right\| \leq i\right\}
$$

is called the $i$-corona about the polytope $P$.

Let us consider a set of pairs $\left(P, \mathscr{C}^{i}(P)\right)$ where $\mathscr{C}^{i}(P)$ is the $i$-corona about the polytope $P$. Two pairs $\left(P, \mathscr{C}^{i}(P)\right)$ and $\left(P^{\prime}, \mathscr{C}^{i}\left(P^{\prime}\right)\right)$ are called equivalent if an isometry $g \in \operatorname{Iso}\left(\mathscr{R}^{d}\right)$ exists that moves both $P$ onto $P^{\prime}$ and $\mathscr{C}^{i}(P)$ onto $\mathscr{C}^{i}\left(P^{\prime}\right)$. 
We pay attention to the fact that there may be two diffrent polytopes $P$ and $P^{\prime}$ that have the same $i$-corona $\mathscr{C}^{i}(P)=\mathscr{C}^{i}\left(P^{\prime}\right)$ for which the pairs $\left(P, \mathscr{C}^{i}(P)\right),\left(P^{\prime}, \mathscr{C}^{i}\left(P^{\prime}\right)\right)$ are not equivalent; namely, when no motion $\tau$ exists with $\tau(P)=P^{\prime}$ and $\tau\left(\mathscr{C}^{i}(P)\right)=\mathscr{C}^{i}\left(P^{\prime}\right)$. Nevertheless, in future we say " $\mathscr{C}(P)$ and $C^{i}\left(P^{\prime}\right)$ are equivalent coronas" by which we mean the equivalence of pairs $\left(P, \mathscr{C}^{i}(P)\right) \sim\left(P^{\prime}, \mathscr{C}^{i}\left(P^{\prime}\right)\right.$.

Given a tiling $T \in \mathscr{T}(\mathscr{P}, \mathscr{R})$ and $k \in \mathbf{N}$, let

$$
\mathscr{U}_{T}^{k}=\left\{U_{i}^{k}\right\}
$$

denote the family of all equivalence classes of $k$-coronas $U_{i}^{k}$ in $T$. Similarly, consider the set of all equivalence classes of $k$-coronas belonging to any of the tilings in the family $\mathscr{T}(\mathscr{P}, \mathscr{R})$. Denote this set by

$$
\mathscr{U}_{\mathscr{T}}^{k}=\bigcup_{T \in \mathscr{T}(\mathscr{P}, \mathscr{R})} \mathscr{U}_{T}^{k}, \quad k \in \mathbf{N}
$$

The following statement is evident.

Lemma 2.1. Let $k \in \mathbf{N}$. The class $\mathscr{U}_{\mathscr{T}}^{k}$ is finite.

Proof. If $k=1$, then the claim is simply the definition of the finiteness of a matching rule. Now assume $\left|\mathscr{U}_{\mathscr{g}}^{k-1}\right|<\infty$. From the finiteness of the matching rule it follows that each tile can be surrounded in only a finite number of possible ways. This implies $\left|\mathscr{U}_{g}^{k}\right|<\infty$.

Given a tiling $T \in \mathscr{T}(\mathscr{P}, \mathscr{R})$. We call an equivalence class $U^{s}$ of $s$-coronas stable with respect to $T$ if the condition $\left(P, \mathscr{C}^{s}(P)\right) \sim\left(P^{\prime}, \mathscr{C}^{s}\left(P^{\prime}\right)\right) \in U^{s} \in \mathscr{U}_{T}^{s}$ implies $\left(P, \mathscr{C}^{k}(P)\right) \sim\left(P^{\prime}, \mathscr{C}^{k}\left(P^{\prime}\right)\right) \in U^{k} \in \mathscr{U}_{T}^{k}$, for all $k>s$.

Lemma 2.2. Let a tiling $T \in \mathscr{T}(\mathscr{P}, \mathscr{R})$ and stable class $U^{s} \in \mathscr{U}_{T}^{s}$. Furthermore, assume we are given two tiles $P$ and $P^{\prime}$ with $\left(P, \mathscr{C}^{s}(P)\right) \in U^{s}$ and $\left(P^{\prime}, \mathscr{C}^{s}\left(P^{\prime}\right)\right) \in U^{s}$. An isometry $\tau \in$ Iso $\mathbf{R}^{d}$ exists such that

$$
\tau:\left\{\begin{array}{l}
P \mapsto P^{\prime}, \\
T \rightarrow T,
\end{array}\right.
$$

i.e., $\tau$ lies in the symmetry group $\Gamma_{T}$ of $T$.

Proof. This fact follows more or less directly from the definition of a stable class. We should mention, however, that not every motion $\tau$, that moves $P$ onto $P^{\prime}$ and $\mathscr{C}^{s}(P)$ onto $\mathscr{C}^{s}\left(P^{\prime}\right)$, necessarily moves $\mathscr{C}^{j}(P)$ onto $\mathscr{C}^{j}\left(P^{\prime}\right)$ with $j>s$ and consequently $T$ is not moved onto itself.

Really, from the stability of the class $U^{s}$ it follows that, for any fixed couple of tiles $P$ and $P^{\prime}$ with $\mathscr{C}^{s}(P) \sim \mathscr{C}^{s}\left(P^{\prime}\right) \subset U^{s}$ and for any $i \geq s$, such a motion $\tau_{i}$ that moves $P$ onto $P^{\prime}$ and $\mathscr{C}^{i}(P)$ onto $\mathscr{C}^{i}\left(P^{\prime}\right)$ exists. The motion $\tau_{s} \tau_{i}^{-1}$ moves both $P$ and $\mathscr{C}^{s}(P)$ onto themselves for every $i \geq s$, or, in other words, the motion $\tau_{s} \tau_{i}^{-1}$ 
belongs to the complete automorphism group Aut $\mathscr{C}_{s}(P)$, which is obviously finite. Therefore in the sequence $\left\{\tau_{s} \tau_{i}^{-1} \mid i \geq s\right\}$ a subsequence consisting of a single motion, say, a motion $\tau$, can be found. The isometry $\tau$ moves $P$ along with its corona $\mathscr{E}^{i}(P)$ of an arbitrarily large rank $i$ onto $\mathscr{C}^{i}\left(P^{\prime}\right)$ with fixed $P^{\prime}$. Hence $\tau$ moves the tiling $T$ onto itself.

Remark. Given two congruent tilings $T$ and $T^{\prime}$ and a stable class $U^{s} \in \mathscr{U}_{T}^{s}$, it is obvious that $U^{s}$ is also present in $T^{\prime}$. However, in principle it is possible that a given stable class $U^{s}$ in a tiling $T$ may be present in a quite different tiling $T^{\prime}$, where it may be a stable class as well as unstable one.

For a fixed tiling $T \in \mathscr{T} \mathscr{P}, \mathscr{R})$ and natural numbers $k, l$ with $k<l$, we write $U^{k} \prec U^{l}$, if a tile $P \in T$ exists such that $\left(P, \mathscr{C}^{k}(P)\right) \in U^{k}$ and $\left(P, \mathscr{C}^{l}(P)\right) \in U^{l}$. Clearly, if $U^{k} \prec U^{l}$ and $U^{l} \nless U^{m}$, then $U^{k} \nless U^{m}$. The relation $\prec$ induces a half-ordering on the set of all classes $\cup_{k=0}^{\infty} \mathscr{L}_{T}^{k}$.

Let us introduce an infinite graph $G_{T}$ with vertex set $\cup_{k=0}^{\infty} \mathscr{Z}_{T}^{k}$. Two vertices (classes) $U_{i}^{k}$ and $U_{j}^{l}$ define an edge of the graph if and only if one follows the other, i.e., either $l=k+1$ and $U_{i}^{k} \prec U_{j}^{l}$, or $k=l+1$ and $U_{i}^{l} \prec U_{j}^{k}$. Each edge $\left(U_{i}^{k}, U_{i}^{k+1}\right)$, with $U_{i}^{k} \prec U_{i}^{k+1}$, is considered to be directed from $U_{i}^{k}$ to $U_{i}^{k+1}$.

Lemma 2.3. The directed graph $G_{T}$ is a finite forest of infinite trees, each tree having its root in the protoset $\mathscr{P}$ (the elements of the latter being considered as equivalence classes of 0-coronas):

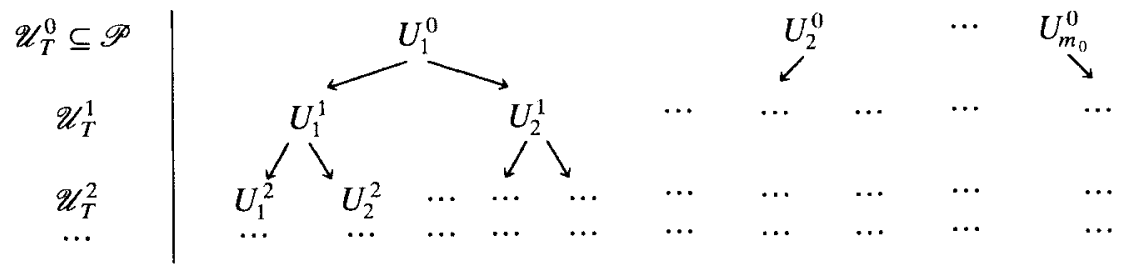

Remark. The tree below any stable class $U_{i}^{s}$ in $G_{T}$ is an infinite chain without any branches.

Lemma 2.4. For any class $U^{k} \in \mathscr{U}_{T}^{k}$ a stable class $U^{s} \in \mathscr{U}_{T}^{s}$ exists such that $U^{k} \prec U^{s}$.

Proof. Before proving this lemma, please note that, although the lemma says that $U^{k}$ has a stable extension $U^{s}: U^{k} \prec U^{s}$, it is of course possible that another extension chain $U^{k} \prec U^{k_{1}} \prec \cdots$ may also exist, which does not contain any stable class.

Assume that a class $U^{k}$ that does not possess a stable extension exists. It follows that, for some $U^{l}$ with $U^{k} \prec U^{l}$, at least two distinct classes $U_{m_{1}}^{l+1}$ and $U_{m_{2}}^{l+1}$ exist such that $U^{l} \prec U_{m_{1}}^{l+1}$ and $U^{l} \prec U_{m_{2}}^{l+1}$. This assumption, however, implies that $U^{k}$ has uncountably many different infinite extension chains like this $U^{k} \prec U^{k+1} \prec$ $\cdots \prec \cdots$. 
Now we show that the assumption contradicts the fact that different tilings in $\mathscr{T}(\mathscr{P}, \mathscr{R})$ are countable. Let us take one of uncountably many extension chains of $U^{k}$ along with its initial part $\mathscr{W}=P \prec U^{1} \prec \cdots \prec U^{k} \prec U^{k+1} \prec \cdots$. It is easy to show that given chain $\mathscr{W}$ we can reconstruct a tiling (let us denote it by $T^{\prime}$ ) in a unique way modulo congruence. Now we need to make two essential remarks. First, though the tiling $T^{\prime}$ has been built up with the help of a chain $\mathscr{W}$ of corona classes of the tiling $T$, the tiling $T^{\prime}$ is not necessarily congruent to $T$. It could be a quite different tiling. On the other hand, two different chains $\mathscr{W}$ and $\mathscr{W}$ can generate the same (or congruent) tiling $T$, as well as different (noncongruent) tilings.

Since by assumption a corona $U^{k} \in \mathscr{U}_{T}^{k}$ does not possess any stable extension, there are uncountably many different sequences $\mathscr{W}$. Some different sequences correspond to sequences of coronas about tiles from noncongruent tilings, other ones can correspond to different tiles from congruent tilings, i.e., tilings of the same class.

As all tiles in countably many different (modulo congruence) tilings are also countable, then all possible infinite extension chains of any corona $U^{k}$ must be at most countable as well.

Lemma 2.5. Let $T \in \mathscr{T}(\mathscr{P}, \mathscr{R})$ be periodic with precisely $m$ different $\Gamma_{T}$ equivalence classes of tiles, i.e., $\left|T / \Gamma_{T}\right|=m$. Then the $(m-1)$-corona $\mathscr{E}^{m-1}(P)$ of any tile $P \in T$ covers a fundamental domain of $\Gamma_{T}$.

Proof. Consider a tile $P \in T$. From the fact that $\left|T / \Gamma_{T}\right|$ is equal to $m$ it follows that a number $k \leq m$ exists such that $\mathscr{C}^{k-1}(P) / \Gamma_{T}=\mathscr{C}^{k}(P) / \Gamma_{T}$, i.e., each tile contained in $\mathscr{C}^{k}(P)$ is $\Gamma_{T}$ equivalent to some tile contained in $\mathscr{C}^{k-1}(P)$. In other words, for every $P^{\prime} \in \mathscr{C}^{k}(P)$ a symmetry $\tau \in \Gamma_{T}$ exists such that $\tau\left(P^{\prime}\right) \in$ $\mathscr{C}^{k-1}(P)$.

We claim that $\mathscr{C}^{k-1}(P)$ covers a fundamental domain. Assume this claim is false. Let $P^{\prime}$ be a tile that is not equivalent to any tile in $\mathscr{C}^{k-1}(P)$ (or even $\mathscr{C}^{k}(P$ ), for that matter) and assume that $P^{\prime}$ is the nearest tile to $P$ among all tiles in $\Gamma_{T} \cdot P^{\prime}$. Then, with $l=\left\|P, P^{\prime}\right\|>k$, clearly $\mathscr{C}^{l-1}(P) \cap \Gamma_{T} \cdot P^{\prime}=\varnothing$. Now connect $P$ and $P^{\prime}$ by a polyhedral chain of the shortest length $P, P_{1}, \ldots, P_{k}, \ldots, P_{l}$ with $P_{k} \in \mathscr{C}^{k}(P)$. By assumption a symmetry $\tau \in \Gamma_{T}$ exists such that $\tau P_{k} \in \mathscr{C}^{k-1}(P)$. Hence $\tau P_{k}$ can be connected to $P$ by a chain whose length is less than $k$. Therefore $P$ can be connected to $\tau P_{l}$ by a chain whose length is less than $l$. This, however, contradicts the assumption that $P_{l}$ was the nearest tile to $P$ among the tiles in $\Gamma_{T} \cdot P_{l}$.

For a fixed tiling $T \in \mathscr{T} \mathscr{P}, \mathscr{R})$ let $\mathscr{P}_{U^{k}}$ denote the following set:

$$
\mathscr{P}_{U^{k}}:=\left\{P \in T \mid\left(P, \mathscr{C}^{k}(P)\right) \in U^{k}, U^{k} \in \mathscr{U}_{T}^{k}\right\} .
$$

We say that $\mathscr{P}_{U^{k}}$ fulfills the q-property for $q \in \mathbf{N}$ if $\cup_{P \in \mathscr{F}_{U k}} \mathscr{C}^{q}(P)=T$. So, by Lemma 2.5 it follows that the orbit $\Gamma_{T} \cdot P$ fulfills the $q$-property for some $q \leq$ $\left|T / \Gamma_{T}\right|-1$.

Lemma 2.6. Given a tiling $T \in \mathscr{T}(\mathscr{P}, \mathscr{R})$ and a stable class $U^{s} \in \mathscr{U}_{T}^{s}$, the tiling $T$ is periodic if and only if $\mathscr{P}_{U^{s}}$ fulfills the q-property for some number $q \in \mathbf{N}$. 
Proof. Let $T$ be a periodic tiling, then for the symmetry group $\Gamma_{T}$ we have $\left|\mathscr{T} / \Gamma_{T}\right|=m<\infty$. Consider a given stable class $U^{s}$. By Lemma 2.2 the set $\mathscr{P}_{U^{s}}$ is an orbit $\Gamma_{T} \cdot P$ of some tile $P \in T$, with $s$-corona $\mathscr{C}^{s}(P) \in U^{s}$. By Lemma 2.5 the subfamily $\mathscr{P}_{U^{s}}$ fulfills the $q$-property with $q \leq m-1$.

Conversely, given a tiling $T$, let $U^{s}$ be an arbitrary stable class. By Lemma 2.2 for any given pair of tiles $P$ and $P^{\prime} \in \mathscr{P}_{U^{s}}$ at least one symmetry $\tau \in \Gamma_{T}$ exists that moves the tile $P$ onto $P^{\prime}$. Let us consider the group $\Gamma$ generated by all such motions, whose existence follows from Lemma 2.2 applied to the stable class $U^{s}$. It is clear that $\Gamma \subset \Gamma_{T}$. As $\mathscr{P}_{U^{s}}$ possesses the $q$-property for a fixed number $q$, i.e., $\cup_{P \in \mathscr{F}_{U^{k}}} \mathscr{C}^{q}(P)=T$, any tile of $T$ Is $\Gamma_{T^{-}}$equivalent to some tile from $\mathscr{C}^{q}(P)$, where $P \in \mathscr{P}_{U^{s}}$. In other words, it covers a fundamental domain.

Conversely, any symmetry $\tau \mid \in \Gamma_{T}$ moves every tile $P$ from $\mathscr{P}_{U^{s}}$ together with its stable corona $\mathscr{C}^{s}(P)$ onto $P^{\prime}$ and also its stable corona $\mathscr{C}^{s}\left(P^{\prime}\right)$.

Assume that $\mathscr{P}_{U^{s}}$ fulfills the $q$-property. Then $\cup_{P \in \mathscr{B}_{y^{s}}} \mathscr{C}^{q}(P)=T$ and by Lemma 2.2 it follows that $\mathscr{C}^{q}(P)$ covers a fundamental domain $T / \Gamma_{T}$. This implies that $\Gamma_{T}$ has a compact fundamental domain.

The following lemma is especially important.

Lemma 2.7. Given a nonperiodic tiling $T \in \mathscr{T}(\mathscr{P}, \mathscr{R})$ and some stable class $U^{s} \in \mathscr{U}_{T}^{s}$, then there is a tiling $T^{\prime} \in \mathscr{T}(\mathscr{P}, \mathscr{R})$ with the following properties:

(1) $U^{s} \notin \mathscr{U}_{T^{\prime}}^{s}$.

(2) If $U^{k} \in \mathscr{U}_{T^{\prime}}^{k}$, then $U^{k} \in \mathscr{U}_{T}^{k}$.

Remark. Lemma 2.7 says that the tiling $T^{\prime}$ contains no corona from the class $U^{s}$, although at the same time all finite coronas of $T^{\prime}$ must be present in $T$.

Proof. Assuming $T$ is nonperiodic it follows from Lemma 2.6 that $\mathscr{P}_{U^{s}}$, which is nonempty, does not fulfill the $q$-property for any $q \in \mathbf{N}$.

Let us consider some given infinite increasing sequence $q_{1}<q_{2}<\cdots$. We can choose $P_{1}$ such that $\mathscr{C}^{q_{1}}\left(P_{1}\right) \cap \mathscr{P}_{U^{s}}=\varnothing$. Then we can choose another tile $P_{2}$ $\left(\neq P_{1}\right)$ such that $\mathscr{C}^{q_{2}}\left(P_{2}\right) \cap \mathscr{P}_{U^{s}}=\varnothing$. By repeating this process we obtain an infinite sequence $S=\left\{P_{1}, P_{2}, \ldots\right\}$ of tiles of the tiling $T$ with $\mathscr{C}^{q_{i}}\left(P_{i}\right) \cap \mathscr{P}_{U^{2}}=\varnothing$ for all $i \in \mathbf{N}$.

The finiteness of the protoset $\mathscr{P}$ implies that we can select an infinite subsequence $S_{0}$ of tiles in $S$ which has the property that all its members are congruent to each other. Now, the finiteness of the equivalence class set $\mathscr{U}_{T}^{1}$ implies that we can select an infinite subsequence $S_{1}$ of tiles in $S_{0}$ which has the property that all pairs $\left(P, \mathscr{C}^{1}(P)\right)$ are equivalent to each other, provided $P \in S_{1}$. Continuing in this way for every fixed $i \in \mathbf{N}$ we obtain a subsequence $S_{i}$ in which all pairs $\left(P, \mathscr{C}^{i}(P)\right)$ are equivalent to each other, provided $P \in S_{i}$. This process can be continued indefinitely. Therefore there is an infinite sequence of classes

$$
U^{0} \prec U^{1} \prec \cdots \prec U^{i} \prec \cdots .
$$

Each class $U^{i}$ is present in the tiling $T$ as the $i$-corona of some tile $P_{i}$, say in $T$. 
We need to check that the sequence (*) generates a well-defined tiling $\bar{T}$. Choose a point in $\mathbf{R}^{d}$ and place a tile $\bar{P}$ at the chosen point. This tile is assumed to be congruent to the centers of coronas from the classes $U^{i}$. Note that it does not matter whether $\bar{P}$ actually coincides with a tile of $T$, or not. After choosing the position of $\bar{P}$, we can move the center $P_{1}$ of the 1 -corona $\mathscr{C}^{1}\left(P_{1}\right)$ to $\bar{P}$, with the help of a suitable isometry $\tau_{1}$. Obviously, the set $\tau_{1}\left(\mathscr{C}^{1}\left(P_{1}\right)\right)$ forms a 1 -corona $\mathscr{C}^{1}(\bar{P})$ about $\bar{P}$. similarly, with the help of a second isometry $\tau_{2}$, we can move $P_{2}$ onto $\bar{P}$ in such a way that $\tau_{2}\left(\mathscr{C}^{1}\left(P_{2}\right)\right)=\mathscr{C}^{1}(\bar{P})$. Clearly, $\tau_{2}\left(\mathscr{C}^{1}\left(P_{2}\right)\right)$ can be considered as the 2 -corona $\mathscr{C}^{2}(\bar{P})$ about $\bar{P}$.

In general, if $\mathscr{C}^{i}(\bar{P})$ has already been constructed, then by an appropriate motion $\tau_{i+1}$ we can move $P_{i+1} \in T$ onto $\bar{P}$ in such a way that $\mathscr{C}^{i}\left(P_{i+1}\right)$ is moved onto $\mathscr{C}^{i}(\bar{P})$. The $(i+1)$-corona $\mathscr{C}^{i+1}(\bar{P})$ is given by $\tau_{i+1}\left(\mathscr{C}^{i+1}\left(P_{i+1}\right)\right)$.

This process can be continued indefinitely and as a result we obtain a tiling $\bar{T}$. It is clear by construction that any given finite corona $\mathscr{C}^{k}\left(\bar{P}^{\prime}\right)$, with $\bar{P}^{\prime} \in \bar{T}$, is congruent to some $k$-corona in $T$, i.e., $\mathscr{U}_{T}^{k} \subset \mathscr{U}_{T}^{k}, k=0,1,2, \ldots$ Moreover, we should remember that the tile $\bar{P}$ and all its coronas $\mathscr{C}^{i}(\bar{P})$ belong to the equivalence classes in $(*)$.

On the other hand, by choice of the sequence $\left\{P_{i} \mid P_{i} \in T, i=0,1, \ldots\right\}$, it follows that the tiling $\bar{T}$ cannot contain any tile $\bar{P}^{\prime \prime}$ whose $s$-corona $\mathscr{C}^{s}\left(\bar{P}^{\prime \prime}\right)$ is equivalent to an $s$-corona contained in the stable class $U^{s} \in \mathscr{U}_{T}^{s}$, in other words, $U^{s} \notin \mathscr{U}_{T}^{s}$. Indeed, assume that $\bar{T}$ is a tiling containing a tile with $\mathscr{C}^{s}(\bar{P}) \in U^{s}$, where $U^{s}$ is a stable class for the tiling $T$. Let $r$ be equal to $\left\|\bar{P}, \bar{P}^{\prime}\right\|$. Then the $i$-corona $\mathscr{E}^{i}(\bar{P})$ contains the corona $\mathscr{C}^{s}\left(P^{\prime}\right)$ inside itself, provided $i \geq r+s$. However, this is impossible because, by choice of the sequence $(*)$, every class consists of coronas that contain no coronas from the stable class $U^{s}$.

Lemma 2.7 implies the following weaker version of Theorem 1.1.

Theorem 2.8. If the cardinality of $\mathscr{T}(\mathscr{P}, \mathscr{R})$ is finite, then $\mathscr{T}(\mathscr{P}, \mathscr{R})$ contains at least one periodic tiling.

Proof. Let $T_{1} \in \mathscr{T}(\mathscr{P}, \mathscr{R})$ be a nonperiodic tiling. Consider a stable class $U^{s_{1}} \in \mathscr{U}_{T_{1}}$. By Lemma 2.7 a tiling $T_{2} \in \mathscr{T}(\mathscr{P}, \mathscr{R})$ exists such that $\mathscr{U}_{T_{2}} \subset \mathscr{U}_{T_{1}}$, but $U^{s_{1}} \notin \mathscr{U}_{T_{2}}$, i.e., $T_{2} \nRightarrow T_{1}$. Assume that $T_{2}$ is also nonperiodic. Now consider a stable class $U^{s_{2}} \in \mathscr{U}_{T_{2}}$. Again, apply Lemma 2.7 to obtain a tiling $T_{3} \in \mathscr{T}(\mathscr{P}, \mathscr{R})$, with $\mathscr{U}_{T_{3}} \subset \mathscr{U}_{T_{2}} \subset \mathscr{U}_{T_{1}}$ and $\left\{U^{s_{1}}, U^{s_{2}}\right\} \not \subset \mathscr{U}_{T_{3}}$. This process can only produce a periodic tiling, since otherwise it would give rise to an infinite sequence of pairwise noncongruent tilings.

Now, in the final part of the paper we must prove Theorem 1.1 for the case $|\mathscr{T}(\mathscr{P}, \mathscr{R})|=\boldsymbol{\aleph}_{0}$. We do this by applying Cantor's diagonalization process.

Assuming that all members of $\mathscr{T}(\mathscr{P}, \mathscr{R})$ are countably many, we can arange them as indicated in Table 1 . We make some comments on this table. In the column "Tilings" we enumerate all tilings in $\mathscr{T} \mathscr{P}, \mathscr{R})$ starting at the top of the table and going downward.

Let $\mathscr{T}_{U^{k}}$ denote a subset of all tilings that contain $k$-coronas from the class $U^{k}$ :

$$
\mathscr{T}_{U^{k}}:=\left\{T \in \mathscr{T}(\mathscr{P}, \mathscr{R}) \mid U^{k} \in \mathscr{U}_{T}^{k}\right\}
$$


Table 1. The diagonalization process.

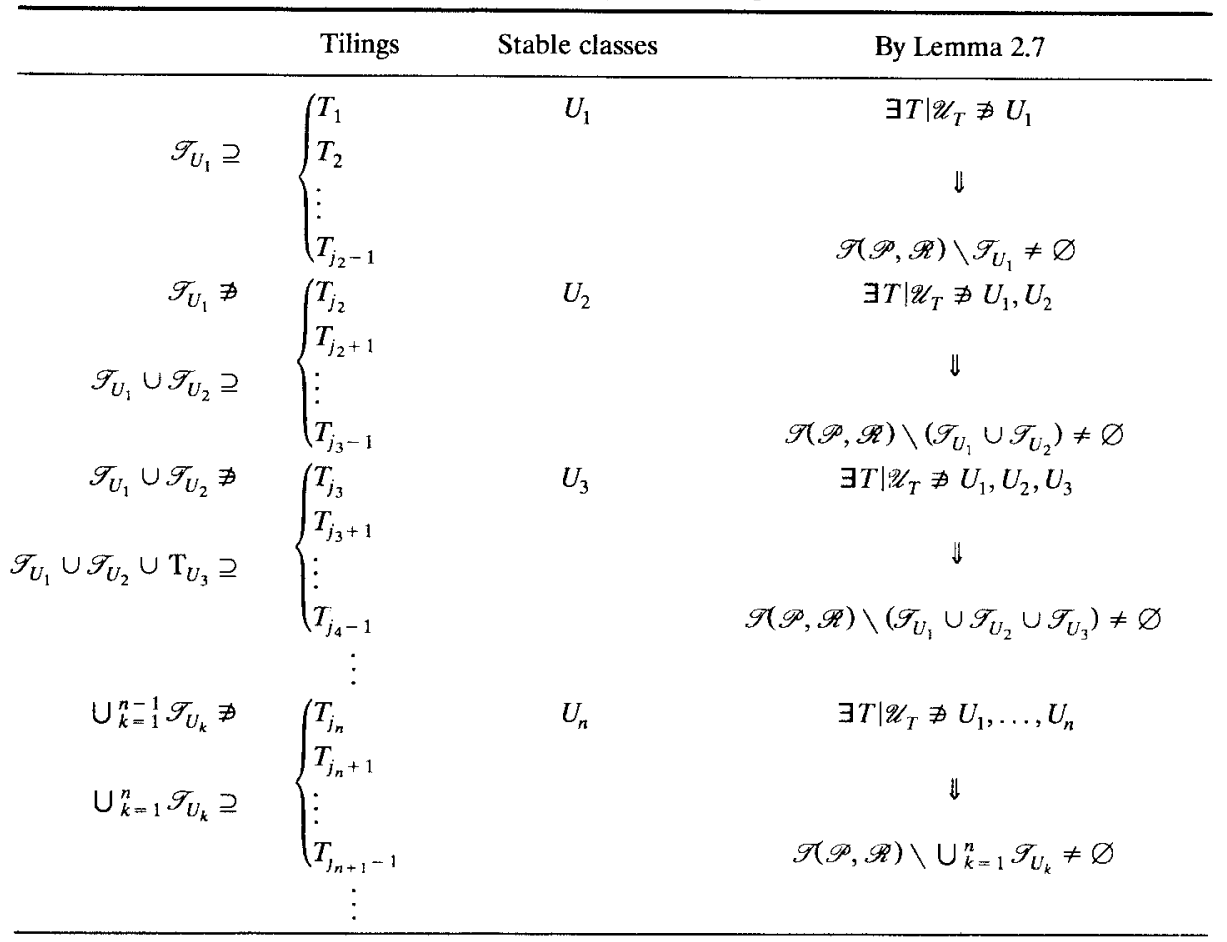

Assume that all members of the list $\mathscr{T}(\mathscr{P}, \mathscr{R})$ are nonperiodic.

Let $T_{1}$ be the first tiling listed and let $U_{1}$ be some stable class for $T_{1}$. All members of the subfamily $\mathscr{T}_{U_{1}}$ have $s_{1}$-coronas from the class $U_{1}$, although the class $U_{1}$ is not necessarily stable for them. On the other hand, Lemma 2.7 implies, for the tiling $T_{1}$ and its fixed stable corona $U_{1}$, that not all tilings in $\left.\mathscr{T} \mathscr{P}, \mathscr{R}\right)$ contain coronas from $U_{1}$, i.e.,

$$
\mathscr{T}(\mathscr{P}, \mathscr{R}) \backslash \mathscr{T}_{U_{1}} \neq \varnothing
$$

Let $T_{j_{2}}$ be the first of the listed tilings that does not belong to $\mathscr{T}_{U_{1}}$, and let $U_{2}$ be some stable class for $T_{j_{2}}$. By applying Lemma 2.7 to $T_{j_{2}}$ and its stable $U_{2}$ we obtain the existence of tilings that do not belong $\mathscr{T}_{U_{1}} \cup \mathscr{T}_{U_{2}}$, i.e., $\mathscr{T}(\mathscr{P}, \mathscr{R}) \backslash\left(\mathscr{T}_{U_{1}} \cup \mathscr{T}_{U_{2}}\right)$ $\neq \varnothing$.

Let $T_{j_{3}}$ be the first in the list of tilings that does not belong to $\mathscr{T}_{U_{1}} \cup \mathscr{T}_{U_{2}}$. Again we obtain the fact that $\mathscr{T}(\mathscr{P}, \mathscr{R}) \backslash\left(\mathscr{T}_{U_{1}} \cup \mathscr{T}_{U_{2}} \cup \mathscr{T}_{U_{3}}\right)$ is not empty.

Since we assume all tilings in $\mathscr{T}(\mathscr{P}, \mathscr{R})$ to be nonperiodic this process obviously must go on indefinitely.

Consider the sequence of the tilings $T_{j_{n}}$ with new notations:

$$
S_{0}:=\left\{\mathbf{T}_{n}: \equiv T_{j_{n}}, n=1,2, \ldots\right\}
$$


For the sequence $S_{0}$ a 1 corona class $U^{1}$ can be found that is present in an infinite number of tilings in the sequence $S_{0}$. Hence we can select an infinite subsequence:

$$
S_{1}:=\left\{\mathbf{T}_{n}\left|\mathbf{T}_{n} \in S_{0}, U^{1} \in \mathscr{U}_{\mathbf{T}_{n}}, n \in \mathbf{N}_{1} \subset \mathbf{N},\right| S_{1}|=| \mathbf{N}_{1} \mid=\mathbf{\aleph}_{0}\right\}
$$

Given $S_{1}$ we can find a 2 -corona class $U^{2}$ with $U^{1} \prec U^{2}$, that is present in infinitely many different tilings in $S_{1}$. Again, we can select an infinite subsequence

$$
S_{2}:=\left\{\mathbf{T}_{n}\left|\mathbf{T}_{n} \in S_{1}, U^{2} \in \mathscr{U}_{\mathbf{T}_{n}}, n \in \mathbf{N}_{2} \subset \mathbf{N},\right| S_{2}|=| \mathbf{N}_{2} \mid=\mathbf{x}_{0}, U^{1} \prec U^{2}\right\} .
$$

Continuing in this way we obtain

$$
S_{k}:=\left\{\mathbf{T}_{n}\left|\mathbf{T}_{n} \in S_{k-1}, U^{k} \in \mathscr{U}_{\mathbf{T}_{n}}, n \in \mathbf{N}_{k} \subset \mathbf{N},\right| S_{k}|=| \mathbf{N}_{k} \mid=\boldsymbol{\aleph}_{0}, U^{k-1} \prec U^{k}\right\}
$$

for any given $k \in \mathbf{N}$.

As shown in the proof of Lemma 2.7, for any given infinite sequence of successively extending classes

$$
U^{1} \prec U^{2} \prec \cdots \prec U^{k} \prec \cdots,
$$

we can construct a tiling $T$ starting with some tile $P \in \mathscr{T}$ in such a way that each $k$-corona $\mathscr{C}^{k}(P)$ lies in a class $U^{k}$.

Finally, to complete the proof of Theorem 1.1, we must make sure that the tiling $T$ does not contain any tile that possesses a corona that lies in one of the classes $U_{n}$, enumerated in Table 1 . Indeed, if such a tile $P^{\prime} \in T$ with a corona $\mathscr{C}^{s}\left(P^{\prime}\right) \in U_{n}$ was to exist, then there would be some number $q_{0}$ such that $\mathscr{C}^{q}\left(P^{\prime}\right) \supset \mathscr{C}^{s}\left(P^{\prime}\right)$, for every $q>q_{0}$. This, however, would imply the existence, in the sequence $S_{0}$, of infinitely many tilings which contain coronas from the class $U_{n}$. However, this is impossible, by choice of the tilings $\mathbf{T}_{1}, \mathbf{T}_{2}, \ldots, \mathbf{T}_{n}, \ldots$.

This contradiction completes the proof of Theorem 1.1.

Remarks. We mention several points that put this result into perspective.

(1) The well-known Penrose tilings in a two-dimensional plane, or the Danzer-Socolar tilings in three-dimensional space [2], present an example of a finite protoset with a finite matching rule that gives rise to an uncountable set of different tilings, none of which is periodic. In contrast to this situation, our result shows that as soon as a given protoset and local rules are such that they generate not more than countably many tilings, then among the tilings at least one must be periodic.

It is well known that the Schmitt-Conway-Danzer (SCD) biprism can fill threedimensional euclidean space only in nonperiodic ways (see [3]). This remarkably simple polyhedron gives an example of a tile that is aperiodic (it is curious that an aperiodic tile in the plane is still unknown). In the beginning the biprism did not have any decoration at its boundary facets and it admitted continuous shifting of two-dimensional layers each with respect to one another. A finite local rule for the biprism only appeared after Danzer had decorated the biprism boundary by some 
specific net and in this way established a finite local rule for the SCD biprism. Nevertheless, since among tilings of space by SCD prisms there are no one periodic tilings, then, despite the well-defined local rule from Theorem 1.1, it follows that there must be uncountably many different tilings of space by the SCD biprisms.

(2) Consider hyperbolic space. In contrast to the euclidean case even in the hyperbolic plane an aperiodic protoset consisting of a single prototile. This fascinating prototile and its corresponding tiling wee discovered, though for a different purpose, by Böröczky [1] and later independently by Shtogrin. Any such tiling can be arranged as a face-to-face tiling having pairwise congruent 1-coronas. It is extremely interesting that, in spite of such strong local conditions, none of the tilings has the symmetry group $\Gamma_{T}$ with compact fundamental domain, i.e., none of them is periodic in the sense of our definition. Moreover, in spite again of such a strong matching rule it gives rise to an uncountable number of different tilings, as is expected from Theorem 1.1.

(3) It is easy to show examples of finite protosets $\mathscr{P}$ and finite matching rules $\mathscr{R}$ which give rise to a finite family of different tilings modulo congruence: $|\mathscr{P}(\mathscr{P}, \mathscr{R})|<$ $\infty$. Among examples of $\mathscr{P}$ and $\mathscr{R}$ with $|\mathscr{T}(\mathscr{P}, \mathscr{R})| \leq \boldsymbol{\kappa}_{0}$, in addition to periodic ones there are also tilings that have no symmetry at all.

\section{Acknowledgments}

I am grateful to the following people for either support or helpful discussions: A. Dress, L. Danzer, M. Shtogrin, D. Huson, and G. Brinkmann. The result has been exposed at several seminars at the Steklov Institute and Moscow University. I am very thankful to the DFG for financal support for my trip to Germany in March 1993 when I had the possibility to discuss the result with my colleagues from the Universities of Bielefeld and Dortmund. I appreciate very much the opportunity to discuss this result at the Regional Geometry Institute at Northampton, MA, and I am grateful to the RGI Advisory Committee and Marjorie Senechal.

\section{References}

1. K. Böröczky, Gömbkitöltések állandó görbületü terekben I, II, Mat. Lapok, 25 (1974), 265-306, 26(1975), 67-90.

2. L. Danzer, Three-dimensional analogs of the planar Penrose tilings and quasicrystals, Discrete Math. 76 (1989), 1-7.

3. M. Senechal, Quasicrystals and Geometry, Cambridge University Press, Cambridge, 1994.

Received February 17, 1994, and in revised form September 30, 1994. 